Check for updates

Cite this: RSC Adv., 2019, 9, 7664

Received 17th December 2018 Accepted 25th February 2019

DOI: $10.1039 / c 8 \mathrm{ra10347j}$

rsc.li/rsc-advances

\section{Structural characterization and anti-inflammatory effect in hepatocytes of a galactoglucan from Antrodia camphorata mycelium $\dagger$}

\author{
Huiling Tang, $\$^{\mathrm{ab}}$ Wenbing Nie, $\neq^{\mathrm{a}}$ Jinna Xiao, ${ }^{\mathrm{a}}$ Zhengqi Zha, ${ }^{\mathrm{a}}$ Qiuli Chen ${ }^{\mathrm{c}}$ \\ and Hongping Yin (D) *a
}

\begin{abstract}
The galactoglucan ACP2 was isolated from cultured Antrodia camphorata mycelium through anionexchange column chromatography and Sephadex G-100 chromatography and shown to exhibit hepatoprotective function in L02 cells. Based on monosaccharide composition analysis, ACP2 was mainly composed of glucose, galactose, and 6-deoxyglucose in a molar ratio of $5: 2: 1$. The average molecular weight of ACP2 was $1.93 \times 10^{4} \mathrm{Da}$. The primary structure of ACP2 was elucidated with Fourier-transform infrared spectroscopy, gas chromatography-mass spectrometry, and nuclear magnetic resonance spectroscopy. The results indicated the following composition: $\rightarrow 6)$-linked- $\beta$-D-

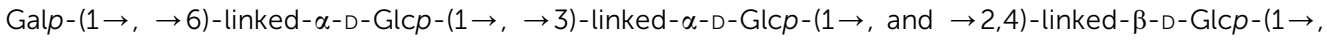
with terminal 6 -deoxy- $\alpha$-D-Glcp and $\alpha$-D-Glcp. ACP2 alleviated lipopolysaccharide-induced hepatocyte inflammation by down-regulating the expressions of COX-2, IL-1 1 , TNF- $\alpha$ and IL- 6 . The decreased expressions of TLR4, MyD88, NF- KB, and phosphorylated p38 in ACP2-treated L02 cells indicated that ACP2 might ameliorate inflammation through the TLR4 and $p 38 / N F-\kappa B$ signaling pathways.
\end{abstract}

\section{Introduction}

Hepatitis, which is caused by various etiological factors such as viral infections, alcoholism, autoimmune disorders and cholestasis, is a complex process that threatens human health and has high incidence. Some studies have found that the pivotal etiology of hepatitis is related to acute liver injury. ${ }^{\mathbf{1 , 2}}$ Some liver injury may be related to lipopolysaccharides (LPS), which are the main component of the cell walls of Gram-negative bacteria and are widely present in the digestive tracts of humans and other animals. ${ }^{3}$ LPS can trigger a series of inflammatory responses and septic shock, eventually leading to multiple organ disorders and liver injury in particular., ${ }^{\mathbf{4} 5}$

Toll-like receptor 4 (TLR4) is a pattern recognition receptor that specially recognizes LPS and initiates signaling cascades, leading to the up-regulation of the expression of pro-

${ }^{a}$ School of Life Science and Technology, State Key Laboratory of Natural Medicines, China Pharmaceutical University, No. 24 Tongjiaxiang Road, Nanjing 210009, Jiangsu, People's Republic of China. E-mail: yinhongping63@163.com; Fax: +86 025 83271249; Tel: +8602583271108

${ }^{b}$ Department of Pharmacy, Jiangsu Food \& Pharmaceutical Science College, Huaian 223003, People's Republic of China

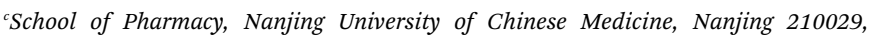
People's Republic of China

$\dagger$ Electronic supplementary information (ESI) available. See DOI: $10.1039 / \mathrm{c} 8 \mathrm{ra} 10347 \mathrm{j}$

$\$$ Both the authors worked equally to this work. inflammatory cytokines. ${ }^{6,7}$ Myeloid differentiation factor 88 (MyD88) is an important adapter protein of TLR4 that can activate mitogen-activated protein kinases (MAPKs) and nuclear factor $-\kappa B(N F-\kappa B)$ signaling pathways. ${ }^{8-10} \mathrm{P} 38$ is a member of the MAPK family that is closely associated with liver inflammation. ${ }^{11} \mathrm{NF}-\kappa \mathrm{B}$ is also a pivotal transcription factor that binds with inhibitors of $\kappa \mathrm{B}(\mathrm{I} \kappa \mathrm{B})$ in the cytoplasm, preventing the transport of NF- $\kappa \mathrm{B}$ into the nucleus. Inflammatory stimuli such as LPS can phosphorylate $\mathrm{I} \kappa \mathrm{B}$ followed by ubiquitination and proteosomal degradation. This leads to the nuclear translocation of NF- $\mathrm{B}$, which promotes the transcription of proinflammatory genes. ${ }^{6}$ In turn, a series of inflammatory factors such as cyclooxygenase-2 (COX-2), tumor necrosis factor- $\alpha$ (TNF$\alpha$ ), interleukin-1 $\beta$ (IL-1 $\beta$ ), and interleukin-6 (IL-6) are upregulated, leading to liver inflammation. ${ }^{12}$

Antrodia camphorata, which is referred to as niu-chang-chih in China, is a parasitic fungus that lives only on the inner cavity walls of the local evergreen Cinnamomum kanchirai Hay in Taiwan. ${ }^{13}$ Among Chinese people, A. camphorata is a valuable traditional functional food that is known as a food supplement or health food for the treatment of hypohepatia and hepatitis. $A$. camphorata exhibits significant hepatoprotection due to its bioactive constituents, which include triterpenoids and ergostane. ${ }^{\mathbf{1 4 , 1 5}}$ However, to the best of our knowledge, the primary structures and hepatoprotective activities of polysaccharides isolated from $A$. camphorata have not been fully investigated. ${ }^{\mathbf{1 6 , 1 7}}$ A member of our research group isolated a water-soluble $\beta$-D- 
glucan from $A$. camphorata and found that it possessed antioxidant activity in human hepatocytes. ${ }^{18}$

Based on a preliminary study of the structures and molecular mechanisms of polysaccharides in our laboratory, we isolated an undescribed galactoglucan termed ACP2 from the mycelium of $A$. camphorata and evaluated its anti-inflammation effect in L02 cells. ACP2 is special because of the presence of 6-deoxyglucose in its sugar chain, which has rarely been reported in the previously papers. We hypothesized that ACP2 attenuates LPSinduced hepatic injury by inhibiting the TLR4-mediated inflammatory pathway and ameliorating the production of downstream pro-inflammatory molecules. The role of 6-deoxyglucan in the anti-inflammatory activity of ACP2 in hepatocytes, if any, requires further study.

\section{Materials and methods}

\subsection{Materials}

Cultured A. camphorata powder was obtained from Jiangsu Shenhua Pharmaceutical Co., Ltd. LPS from Escherichia coli (O55:B5, L2880) and standard monosaccharides were provided by Sigma. A series of glucan standards with different molecular weights were obtained from Shodex. Methyl-thiazolyltetrazolium (MTT), which was used for coloration, was purchased from Invitrogen Corp. (Carlsbad, CA, USA). Dulbecco's modified eagle medium (DMEM) and fetal bovine serum were purchased from Gibco (Rockville, MD, USA), and polyvinylidene difluoride (PVDF) membranes were purchased from Bio-Rad (Hercules, CA, USA). All other chemicals and solvents were analytical reagent grade.

\subsection{Extraction and purification}

ACP2 was extracted and purified according to the methods of Wang. ${ }^{19}$ Briefly, after a series of steps involving dissolving, decolorizing, purifying and drying, we obtained decolorized polysaccharide (dACP). The dACP was separated through a column $(300 \times 26 \mathrm{~mm})$ packed with diethylaminoethyl (DEAE)-cellulose 52 and eluted with distilled water followed by $1 \mathrm{~mol} \mathrm{~L}^{-1} \mathrm{NaCl}$. Subsequently, the eluate was collected and purified using a Sephadex G-100 column $(1.0 \times 80 \mathrm{~cm})$ followed by dialysis and lyophilization in a vacuum freeze-dryer. The resulting depurated polysaccharide fraction was assigned as ACP2. The purity of ACP2 was preliminarily analyzed using ultraviolet (UV) spectroscopy.

\subsection{Determination of molecular weight}

The molecular weight of ACP2 was estimated by highperformance gel-permeation chromatography (HPGPC) with a refractive index detector (detection temperature $=35^{\circ} \mathrm{C}$ ) and Shodex SB 803 and Shodex SB 805 chromatographic columns. The detailed experimental conditions were as follows: the sample solution $(100 \mu \mathrm{L})$ was injected and eluted with $0.1 \mathrm{M}$ $\mathrm{NaNO}_{3}$ solution at a flow rate of $0.35 \mathrm{~mL} \mathrm{~min}{ }^{-1} \cdot{ }^{20}$ The calibration curve was established using GPC software.

\subsection{Monosaccharide composition analysis of ACP2}

High-performance liquid chromatography (HPLC) was used to identify the monosaccharide composition of ACP2 based on 1phenyl-3-methyl-5-pyrazolone (PMP) labeling. ${ }^{21}$ Briefly, ACP2 solution ( $5 \mathrm{mg} \mathrm{mL}^{-1}$ ) was hydrolyzed by $2 \mathrm{M}$ trifluoroacetic acid (TFA) for $2 \mathrm{~h}$ at $100{ }^{\circ} \mathrm{C}$. After removing TFA, the hydrolysate was derivatized via the addition of $0.6 \mathrm{M} \mathrm{NaOH}(25 \mu \mathrm{L})$ and $0.4 \mathrm{M}$ PMP $(50 \mu \mathrm{L})$. Subsequently, the solution was neutralized with 50 $\mu \mathrm{L}$ of $0.3 \mathrm{M} \mathrm{HCl}$ and extracted three times with chloroform. The aqueous phase was then loaded onto an HPLC using an Agilent 1260 system (UV detector VWD, wave length $250 \mathrm{~nm}$ ) equipped with a ZORBAX Eclipse XDB-C18 $(250 \mathrm{~mm} \times 4.6 \mathrm{~mm})$ column and eluted at $0.8 \mathrm{~mL} \mathrm{~min}^{-1}$ with acetonitrile and phosphate buffer (pH 6.7) in a ratio of $17: 83(\mathrm{v} / \mathrm{v})$.

\subsection{Fourier-transform infrared (FT-IR) spectroscopy}

ACP2 was analyzed on a FT-IR spectrophotometer (Bruker Tensor 27, Bruker, Germany). Briefly, the sample was ground with dried $\mathrm{KBr}$ powder and then compressed into a $1 \mathrm{~mm}$ pellet for analysis in the frequency range of $4000-400 \mathrm{~cm}^{-1}$.

\subsection{Nuclear magnetic resonance (NMR) spectroscopy}

ACP2 samples were dissolved in $1.0 \mathrm{~mL} \mathrm{D}_{2} \mathrm{O}$. NMR spectra were recorded at $25^{\circ} \mathrm{C}$ using a JEOL JNM ECP 600 NMR spectrometer (JEOL, Tokyo, Japan). ${ }^{1} \mathrm{D}\left({ }^{1} \mathrm{H}\right.$ and ${ }^{13} \mathrm{C}$ NMR) and ${ }^{2} \mathrm{D}$ NMR spectra, ${ }^{1} \mathrm{H}^{-1} \mathrm{H}$ correlated $\left({ }^{1} \mathrm{H}^{-}{ }^{1} \mathrm{H} \mathrm{COSY}\right)$ spectra, heteronuclear single quantum correlation (HSQC) spectra, and heteronuclear multiple bond correlation (HMBC) spectra were collected.

\subsection{Methylation analysis}

ACP2 was methylated repeatedly in accordance with Ciucanu's method. ${ }^{22}$ The absolute methylated products were hydrolyzed with 2 M TFA and converted into the partially methylated alditol acetates (PMAAs) that were analyzed by using a gas chromatography-mass spectrometry (GC-MS; HP 6890 II, Agilent USA) equipped with a DB-225MS fused-silica capillary column (Agilent Technologies Co. Ltd., USA).

\subsection{Biological activity}

2.8.1. Cell culture. Human L02 liver cell line (L02) was obtained from the Cell Resource Center of the Institutes of Biomedical Sciences, Fudan University. Cells were cultured in DMEM medium supplemented with $10 \%$ fetal bovine serum and $1 \%$ penicillin/streptomycin at $37{ }^{\circ} \mathrm{C}$ in a humidified incubator with atmosphere containing $5 \% \mathrm{CO}_{2}$.

2.8.2. Cell proliferation viability assay. Cell viability was determined by MTT assay as follows: ${ }^{23}$ L02 cells $\left(2 \times 10^{4}\right.$ cells per well) were plated in 96-well plates overnight, and different concentrations of ACP2, LPS, and glutathione (GSH) were added to the wells. Following this incubation, $20 \mu \mathrm{L}$ of $5 \mathrm{mg} \mathrm{mL}^{-1}$ MTT was added into each well and incubated for an additional $4 \mathrm{~h}$. Afterwards, the supernatant was completely replaced with 150 $\mu \mathrm{L}$ of DMSO and purple color crystals were dissolved. Finally, the absorbance was then evaluated at $570 \mathrm{~nm}$ using a Microplate reader (BioTek, USA). 
2.8.3. Western blot assay. L02 cells were harvested and suspended in RIPA lysis buffer. Extracted total cellular proteins were separated on a $12 \%$ sodium dodecyl sulfatepolyacrylamide gel and transferred onto polyvinylidene fluoride (PVDF) membranes. The membranes were incubated overnight with primary antibody at $4{ }^{\circ} \mathrm{C}$. After washing three times in Tris-buffered saline and Tween 20 (TBST), the membranes were incubated with goat anti-rabbit IgG secondary antibody for $1 \mathrm{~h}$ at room temperature. Finally, the expressions of different proteins were visualized using an enhanced chemiluminescence system (MILIPORE, USA). The antibodies used for western blot analysis were as follows: TLR4, MyD88, NF- $\kappa \mathrm{B}$, IкB- $\alpha$, p38, p-p38, JNK, p-JNK, ERK, and p-ERK (Cell Signaling Technology, USA); IL-1 $\beta$, IL-6, COX-2 and TNF- $\alpha$ (Sangon Biotech, Shanghai, China); and $\beta$-actin and tubulin (Abcam, UK).

\subsection{Statistical analysis}

All experimental data are presented as means \pm standard deviations. Statistical comparisons were made using Graphpad Prism 5.0 software. Two levels of statistical significance were considered: $* p<0.05$ and ${ }^{* *} p<0.01$.

\section{Results and discussion}

\subsection{Extraction, purification, and characterization of ACP2}

ACP2 was obtained from the mycelium of A. camphorata in a single symmetrical chromatographic peak after a series of purification steps using DEAE-52 and Sephadex G-100 columns. Hydrolyzed ACP2 had no absorption at either $260 \mathrm{~nm}$ or $280 \mathrm{~nm}$ in the UV spectrum, which indicated the absence of proteins and nucleic acids. The HPGPC profile of ACP2 exhibited a single, symmetrical sharp peak (Fig. 1A), which is characteristic of a homogeneous polysaccharide. Based on standard curve and retention time of the polysaccharide (Fig. 1B), the average molecular weight of ACP2 was estimated to be $1.93 \times 10^{4} \mathrm{Da}$.

\subsection{Structural characterization}

3.2.1. Monosaccharide composition. Hydrolysate of ACP2 appeared three single peaks in HPLC (Fig. 1C). Based on the comparison of retention time and peak area between ACP2 and standard monosaccharides, ACP2 consisted of glucose, galactose and 6-deoxyglucose in a molar ratio of $5: 2: 1$ (Fig. 1D).

3.2.2. FT-IR spectroscopy. As shown in ESI $1, \dagger$ the infrared spectrum of purified ACP2 exhibited a strong and broad absorption peak in the range of $4000-400 \mathrm{~cm}^{-1}$, which is a typical feature of polysaccharides. The broadly stretched, intense absorption peaks at $3421.66 \mathrm{~cm}^{-1}$ and $3131.94 \mathrm{~cm}^{-1}$ were attributed to $\mathrm{O}-\mathrm{H}$ stretching vibration. ${ }^{24}$ The weak signals at 2928.93 and $2848.59 \mathrm{~cm}^{-1}$ can be assigned to the stretching vibration of the $\mathrm{C}-\mathrm{H}$ bond of $\mathrm{CH}_{2}$, while the absorption band at $1637.60 \mathrm{~cm}^{-1}$ was attributed to the characteristic peak of bound water. ${ }^{25}$ The characteristic signals between 1200 and $1000 \mathrm{~cm}^{-1}$ were assigned to the stretching vibrations of the $\mathrm{C}-\mathrm{OH}$ side groups and $\mathrm{C}-\mathrm{O}-\mathrm{C}$ glycosidic bond vibration. The absence of the peaks at 810 and $870 \mathrm{~cm}^{-1}$ indicate the absence of mannose. The weak absorption peaks at 837.36 and $886.50 \mathrm{~cm}^{-1}$ reveal the presence of $\alpha$ and $\beta$ epimers in ACP2. ${ }^{26}$

3.2.3. Methylation and GC-MS analysis of ACP2. Methylation and GC-MS analysis were performed to reveal the details structure of ACP2. There were six main alditol acetate derivatives from the methylated detected by GC-MS. As summarized in Table 1. ACP2 showed six derivatives, 2,3,4-Me $\mathrm{Me}_{3}$-6-deoxy-Glc $p$,

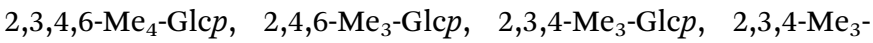

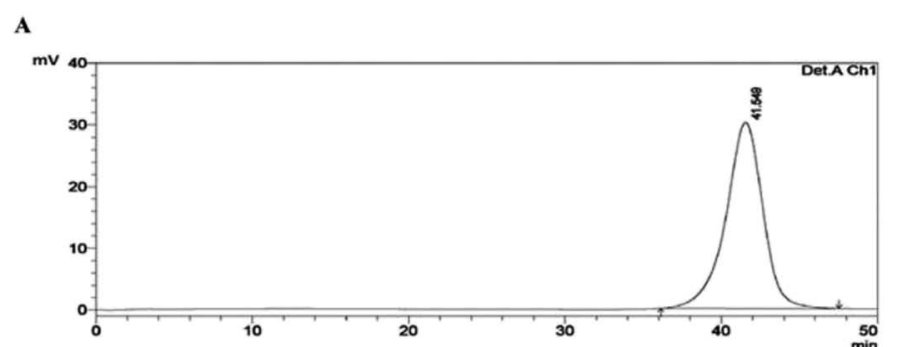

C
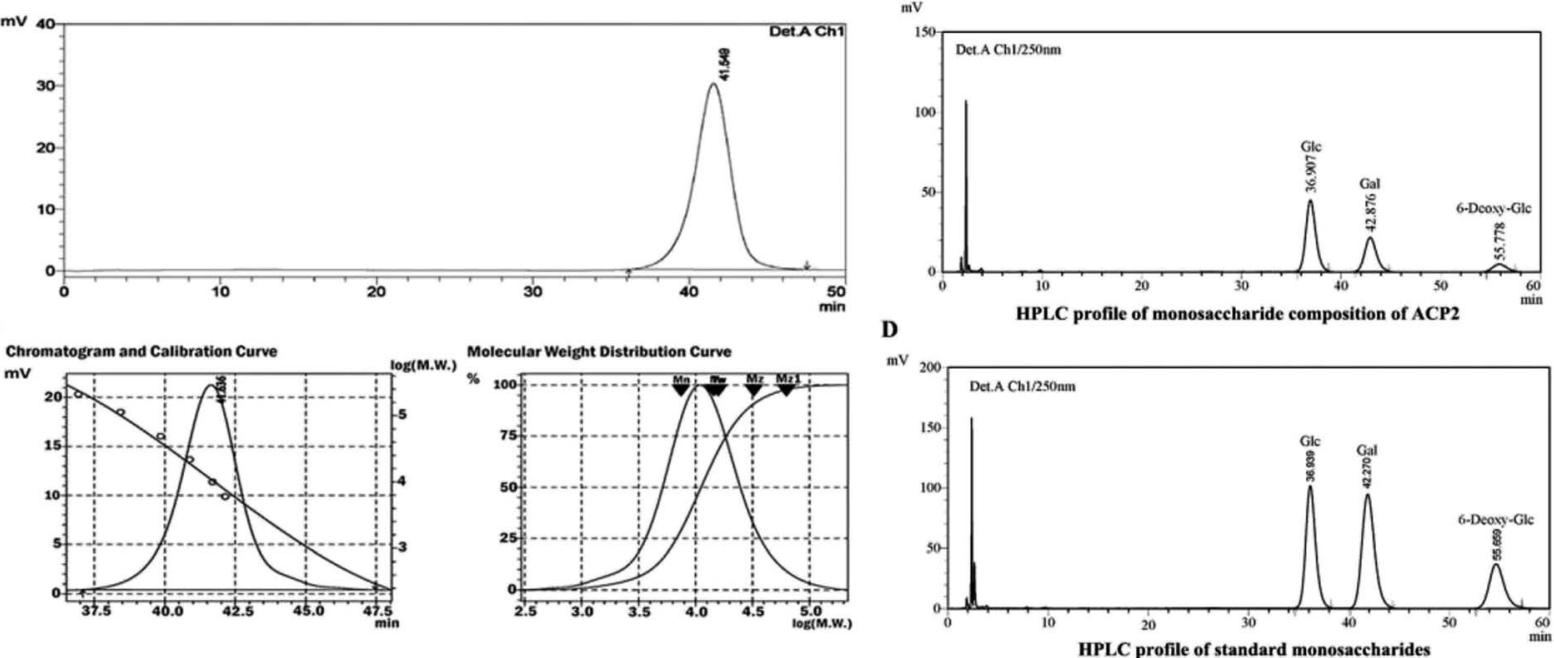

Fig. 1 Elution profile of ACP2 in HPGPC with $0.1 \mathrm{M} \mathrm{NaNO}_{3}$ at a flow rate of $0.35 \mathrm{~mL} \mathrm{~min}^{-1}$ on Shodex SB805 and 802 columns. (A) ACP2 was identified as a single symmetrical sharp peak, indicating that it was a homogeneous polysaccharide. (B) Chromatogram and calibration curve of Dextran standards plotted with GPC software and molecular weight $\left(M_{w}\right)$ on a log scale versus the retention time of ACP2. (C) Monosaccharide composition of ACP2. (D) HPLC profile of standard monosaccharides. 
Table 1 GC-MS data for the main alditol acetate derivatives from the methylated products of ACP-2

\begin{tabular}{lll}
\hline Methylated sugars (as alditol acetates) & Type of linkage & Mass fragments $(\mathrm{m} / \mathrm{z})($ relative abundance, \%) \\
\hline $2,3,4-\mathrm{Me}_{3}-6$-deoxy-Glcp & T-6-deoxy-Glc & $43,59,71,72,89,101,115,117,131,161,175$ \\
$2,3,4,6-\mathrm{Me}_{4}$-Glcp $p^{a}$ & T-Glc & $43,58,59,71,75,87,101,117,129,145,161,205$ \\
$2,4,6-\mathrm{Me}_{3}-\mathrm{Glc} p$ & $1,3-L i n k e d-G l c$ & $43,45,71,87,101,117,129,161,189,233$ \\
$2,3,4-\mathrm{Me}_{3}-\mathrm{Glc} p$ & $1,6-L i n k e d-G l c$ & $43,45,58,71,87,101,117,129,161,173,189,233$ \\
$2,3,4-\mathrm{Me}_{3}-\mathrm{Gal} p$ & $1,6-L i n k e d-G a l$ & $43,45,58,71,87,101,117,129,161,173,189,233$ \\
$3,6-\mathrm{Me}_{2}-\mathrm{Glc} p$ & $1,2,4-L i n k e d-G l c$ & $43,45,71,87,99,113,129,159,189,233$
\end{tabular}

${ }^{a}$ 2,3,4,6- $\mathrm{Me}_{4}$-Glcp = 1,5-di-O-acetyl-2,3,4,6-tetra-O-methyl-glucopyranose, etc.

A

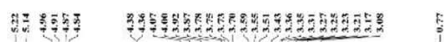
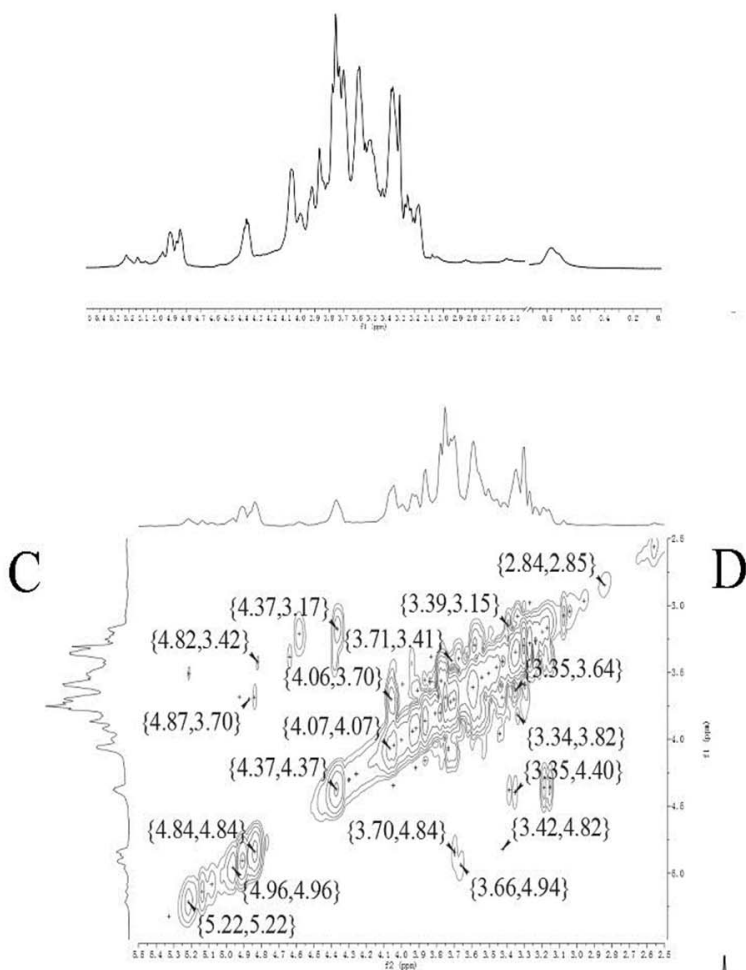

B
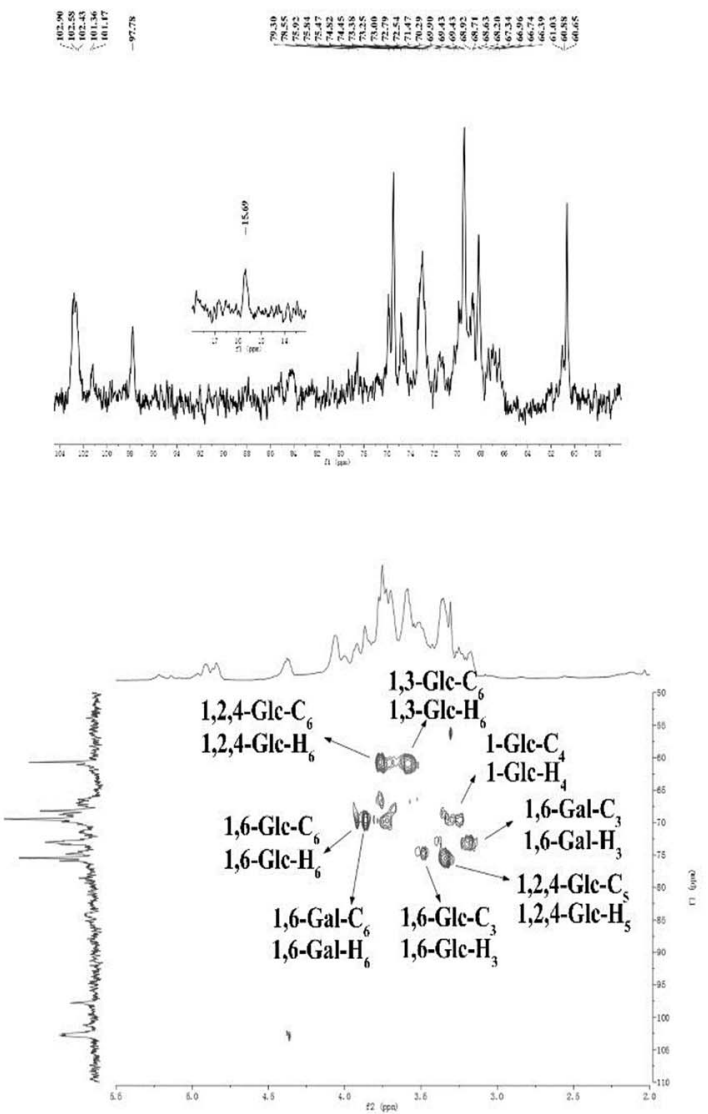

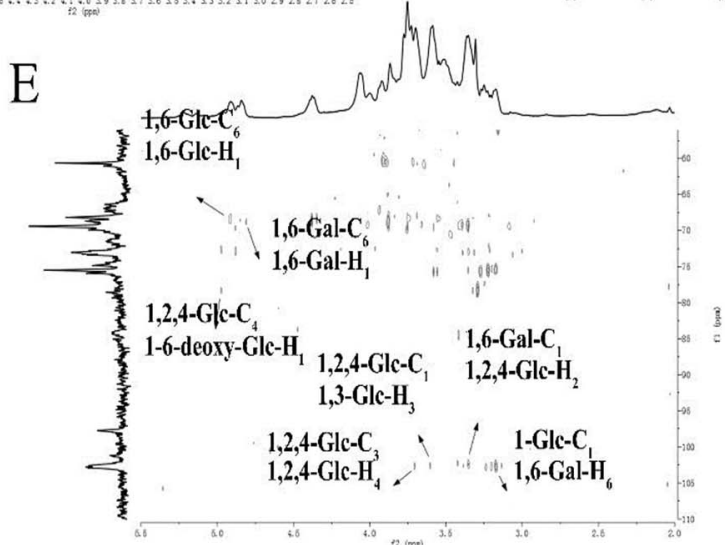

Fig. 2 NMR spectra of ACP2 in $D_{2} \mathrm{O}$. (A) ${ }^{1} \mathrm{H}$ NMR spectrum. (B) ${ }^{13} \mathrm{C}$ NMR spectrum. (C) ${ }^{1} \mathrm{H}-{ }^{1} \mathrm{H}$ COSY spectrum. (D) HSQC spectrum. (E) HMBC spectrum. 
Gal $p$ and 3,6- $\mathrm{Me}_{2}$-Glcp. The results indicated that the main chain was composed of 1,3-linked-Glc, 1,6-linked-Glc, 1,2,4linked-Glc. The branched structure in ACP2 appeared in 1,2,4linked-Glc, whose C-4 was attached to T-6-deoxy-Glc, meanwhile the C-2 was attached to 1,6-linked-Gal; C- 6 of galactose was attached to terminal glucose as shown in ESI 2. $\dagger$ The repeating segments were indicated to form the whole ACP2 molecule.

3.2.4. NMR spectroscopy analysis of ACP2. The NMR spectrum of ACP2 (Fig. 2A) exhibited six anomeric proton signals with chemical shifts of $\delta \mathrm{H} \mathrm{5.22,} \mathrm{5.14,} \mathrm{4.96,} \mathrm{4.91,} 4.87$ and $4.84 \mathrm{ppm}$. These signals indicate the presence of both the $\alpha$ anomeric and $\beta$-anomeric configurations. ${ }^{25}$ The ${ }^{13} \mathrm{C}$ NMR spectra of ACP2 are shown in Fig. 2B, and the chemical shift data are provided in Table 2.

The correlations between hydrogen and carbon atoms were determined from the ${ }^{1} \mathrm{H}^{1}{ }^{1} \mathrm{H}$ COSY and HSQC spectra (Fig. 2C and $\mathrm{D}$, respectively). ${ }^{27}$ The signals at $\delta \mathrm{H} \mathrm{3.78,4.36/ \delta} \mathrm{C} 60.65$ were attributed to H-6/C- 6 of $\rightarrow 2,4)-\beta$-D-Glc $p$ - $(1 \rightarrow$, and the

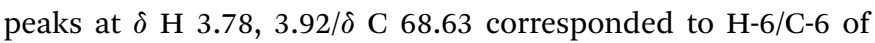

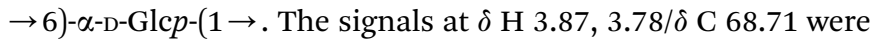
attributed to H-6/C- 6 of $\rightarrow 6)-\beta$-D-Gal $p-(1 \rightarrow$. The peaks at $\delta \mathrm{H}$ $3.43 / \delta \mathrm{C} 74.45, \delta \mathrm{H} 3.35 / \delta \mathrm{C} 74.47$, and $\delta \mathrm{H} 3.87 / \delta \mathrm{C} 73.25$ were assigned to $\mathrm{H}-3 / \mathrm{C}-3$ of $\rightarrow 6)-\beta$-D-Glc $p$ - $(1 \rightarrow, \mathrm{H}-5 / \mathrm{C}-5$ of $\rightarrow 2,4)-\beta-\mathrm{D}-$ Glc $p-(1 \rightarrow$, and $\mathrm{H}-3 / \mathrm{C}-3$ of $\rightarrow 6)-\beta-\mathrm{D}-\mathrm{Gal} p-(1 \rightarrow$, respectively. The

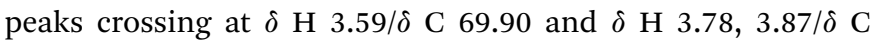
60.880 were attributed to $\mathrm{H}-4 / \mathrm{C}-4$ of $\alpha$-D-Glc $p$ - $(1 \rightarrow$ and $\mathrm{H}-3 / \mathrm{C}-3$ of $\rightarrow 3)-\alpha$-D-Glc $p$ - $(1 \rightarrow$.

The HMBC spectrum indicates both the remote coupling correlation between carbon and hydrogen along with the corresponding position of the glycosidic linkage. As shown in Fig. 2E, the signals at $\delta \mathrm{H} 4.91 / \delta \mathrm{C} 68.63 \mathrm{ppm}$ were attributed to H-1 of $\rightarrow 6)-\alpha$-D-Glc $p$ - $(1 \rightarrow$ and C- 6 of $\rightarrow 6)-\alpha-D-G l c p-(1 \rightarrow$, and the peaks at $\delta \mathrm{H} \mathrm{4.81/ \delta} \mathrm{C} 68.71 \mathrm{ppm}$ were speculated to correspond to $\mathrm{H}-1$ of $\rightarrow 6)-\beta$-D-Gal $p$ - $(1 \rightarrow$ and C- 6 of $\rightarrow 6)-\beta$-D-Gal $p$ $(1 \rightarrow$. The signals at $\delta \mathrm{H} 4.96 / \delta \mathrm{C} 78.55 \mathrm{ppm}$ were assigned to $\mathrm{H}-1$ of 6-deoxy- $\alpha$-D-Glc $p$ - $(1 \rightarrow$ and C- 4 of $\rightarrow 2,4)-\beta$-D-Glc $p$ - $(1 \rightarrow$, while the peaks at $\delta \mathrm{H} 3.70 / \delta \mathrm{C} 102.58 \mathrm{ppm}$ attributed to $\mathrm{H}-4$ of $\rightarrow 2,4)-$ $\beta$-D-Glc $p-(1 \rightarrow$ and C- 1 of $\rightarrow 2,4)-\beta$-D-Glc $p-(1 \rightarrow$. The signals at $\delta \mathrm{H} 3.59 / \delta \mathrm{C} 102.58$ ppm were assigned to $\mathrm{H}-3$ of $\rightarrow 3)-\alpha-\mathrm{D}-\mathrm{Glc} p$ -

Table $2{ }^{13} \mathrm{C}$ and ${ }^{1} \mathrm{H}$ NMR chemical shifts of the main residues from $\mathrm{ACP} 2$ in $\mathrm{D}_{2} \mathrm{O}$

$$
\delta{ }^{13} \mathrm{C} /{ }^{1} \mathrm{H}(\mathrm{ppm})
$$

\begin{tabular}{|c|c|c|c|c|c|c|}
\hline Sugar residues & 1 & 2 & 3 & 4 & 5 & 6 \\
\hline \multirow{2}{*}{ 6-Deoxy- $\alpha-\mathrm{D}-\mathrm{Glc} p-(1 \rightarrow$} & 102.90 & 71.47 & 73.25 & 68.97 & 75.47 & 15.69 \\
\hline & 4.96 & 3.35 & 3.51 & 3.08 & 3.75 & 0.77 \\
\hline \multirow[t]{2}{*}{$\alpha$-D-Glcp- $(1 \rightarrow$} & 101.36 & 74.82 & 73.38 & 69.90 & 75.84 & 60.88 \\
\hline & 5.22 & 3.36 & 3.70 & 3.59 & 3.73 & $3.78 / 3.87$ \\
\hline \multirow[t]{2}{*}{$\rightarrow 3)-\alpha-\mathrm{D}-\mathrm{Glc} p-(1 \rightarrow$} & 97.78 & 74.82 & 73.38 & 69.43 & 75.84 & 60.88 \\
\hline & 5.14 & 3.78 & 3.59 & 3.31 & 3.75 & $3.78 / 3.87$ \\
\hline \multirow[t]{2}{*}{$\rightarrow 6)-\alpha-\mathrm{D}-\mathrm{Glc} p-(1 \rightarrow$} & 102.43 & 74.82 & 74.45 & 69.90 & 75.84 & 68.63 \\
\hline & 4.91 & 3.78 & 3.43 & 3.25 & 3.70 & $3.78 / 3.92$ \\
\hline \multirow[t]{2}{*}{$\rightarrow 6)-\beta-\mathrm{D}-\mathrm{Gal} p-(1 \rightarrow$} & 102.43 & 72.54 & 72.79 & 68.71 & 68.92 & 68.71 \\
\hline & 4.84 & 3.17 & 3.87 & 4.07 & 4.38 & $3.87 / 3.78$ \\
\hline \multirow[t]{2}{*}{$\rightarrow 2,4)-\beta$-D-Glcp-(1 $\rightarrow$} & 102.58 & 79.30 & 75.47 & 78.55 & 75.47 & 60.65 \\
\hline & 4.87 & 3.35 & 3.87 & 3.70 & 3.35 & $3.78 / 4.36$ \\
\hline
\end{tabular}

$(1 \rightarrow$ and C- 1 of $\rightarrow 2,4)-\beta$-D-Glcp- $(1 \rightarrow$, whereas the signals at $\delta \mathrm{H}$ $3.35 / \delta$ C 102.43 ppm were attributed to $\mathrm{H}-2$ of $\rightarrow 2,4)-\beta$-D-Glcp$(1 \rightarrow$ and $\mathrm{C}-1$ of $\rightarrow 6)-\beta$-D-Gal $p$ - $(1 \rightarrow$. The signals at $\delta \mathrm{H} \mathrm{3.78/ \delta} \mathrm{C}$ 101.36 ppm corresponded to H- 6 of $\rightarrow 6)-\beta-\mathrm{D}-\mathrm{Gal} p-(1 \rightarrow$ and $\mathrm{C}-1$ of $\alpha$-D-Glc $p-\left(1 \rightarrow\right.$. All ${ }^{13} \mathrm{C}$ NMR chemical shifts are shown in Table 2.

In consideration of the monosaccharide composition, FT-IR spectroscopy, GC-MS, and ${ }^{1} \mathrm{H} /{ }^{13} \mathrm{C}$ NMR spectroscopy analyses, the likely backbone of ACP2 is as follows: $\rightarrow 6)$-linked- $\beta$-D-Gal $p$ $(1 \rightarrow, \rightarrow 6)$-linked- $\alpha$-D-Gcl $p$ - $(1 \rightarrow, \rightarrow 3)$-linked- $\alpha$-D-Glc $p$ - $(1 \rightarrow$, and $\rightarrow 2,4)$-linked- $\beta$-D-Glc $p$ - $(1 \rightarrow$, with terminal 6-deoxy- $\alpha$-D-Glc $p$ and $\alpha$-D-Glcp.

\subsection{Protective effect of ACP2 on $\mathrm{LO2}$ cells}

Prior to determining the anti-inflammatory activity of ACP2, we examined the cytotoxicity of LPS, GSH and ACP2 in L02 cells using MTT assay. As shown in Fig. 3A, LPS significantly inhibited the proliferation of L02 cells at the concentration of $10 \mu \mathrm{g} \mathrm{mL}{ }^{-1}\left({ }^{\# \#} p<0.01\right)$. GSH, a common reducing substance with good hepatoprotection, was used as a positive control in this study. However, GSH inhibited the growth of L02 cells when the concentration reached $15 \mathrm{mM}$ (Fig. 3B); the optimal concentration of GSH was $10 \mathrm{mM}$. As shown in Fig. 3C, ACP2 had no obvious toxic effect on L02 cells until its concentration reached $200 \mu \mathrm{g} \mathrm{mL}^{-1}\left({ }^{\# \#} p<0.01\right)$. The data also indicated a protective effect of ACP2 compared to LPS as concentrations of 25, 50 and $100 \mu \mathrm{g} \mathrm{mL}{ }^{-1}$. Therefore, ACP2 promoted the proliferation of L02 cells. In subsequent tests, we evaluated ACP2 concentrations of 25 and $100 \mu \mathrm{g} \mathrm{mL}{ }^{-1}$, the $\mathrm{GSH}$ concentration of $10 \mathrm{mM}$, and the LPS concentration of $10 \mu \mathrm{g}$ $\mathrm{mL}^{-1}$ (Fig. 3D).

\subsection{ACP2 inhibited TLR4/NF- $\mathrm{B}$ signal pathway}

Inflammation is an intricate pathophysiological phenomenon that is thought to depend on some pathogen recognition receptors such as $\mathrm{TLRs}^{28}{ }^{28}$ which play a pivotal role in liver injury. ${ }^{5}$ Recent studies have demonstrated the presence of TLRs in different cell types, including macrophages, dendritic cells and hepatocytes. ${ }^{29}$ Given that the mass of hepatic cells in the liver, therefore, TLRs can be responsive to various pathogens. MyD88, a signaling adaptor that can be activated by TLRs, plays a crucial role in various biological and pathological processes in hepatocytes. MyD88 triggers the secretion of inflammatory factors that regulate and amplify inflammatory response through TLR signals. ${ }^{30}$ To determine if ACP2 regulates the TLR4-MyD88 signaling pathway in hepatic cells, we evaluated the protein levels of TLR4 and MyD88 in L02 cells. As shown in Fig. 4A-C, compared to the control group, the expressions of TLR4 and MyD88 were significantly up-regulated by treatment with LPS $\left({ }^{\# \#} p<0.01\right)$, while ACP2 treatment clearly downregulated TLR4 and MyD88 at the concentration of $100 \mu \mathrm{g}$ $\mathrm{mL}^{-1}\left({ }^{\# \#} p<0.01\right)$. These results imply that the antiinflammatory effect of ACP2 is related to the TLR4-MyD88 signaling pathway.

Numerous studies have shown that NF- $\kappa \mathrm{B}$ and MAPKs are two downstream effectors of TLR4-MyD88 signaling that 
A
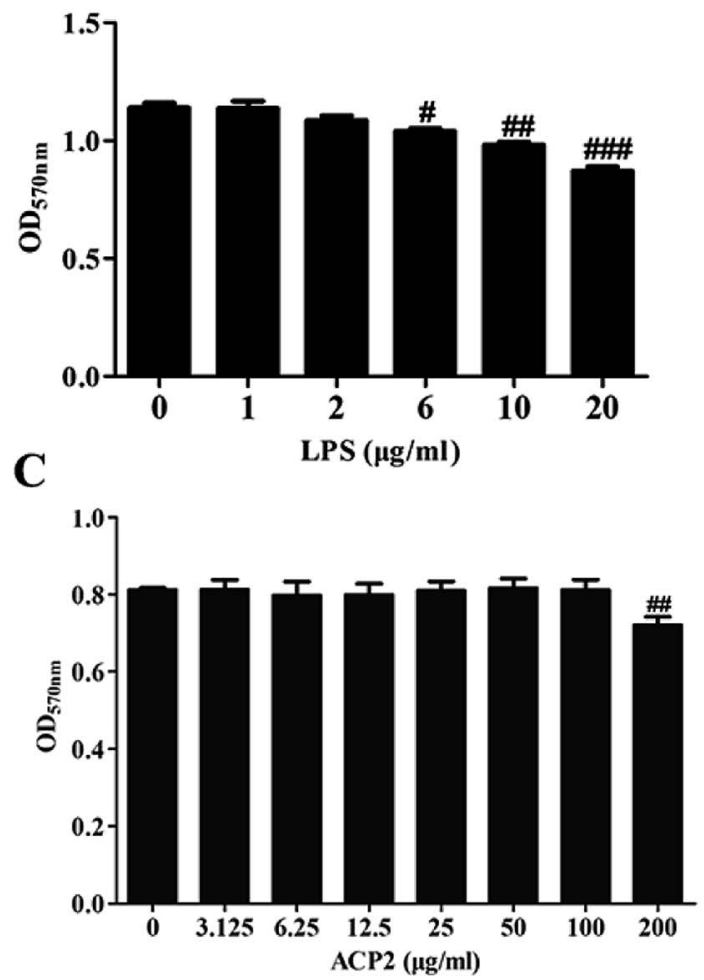

B
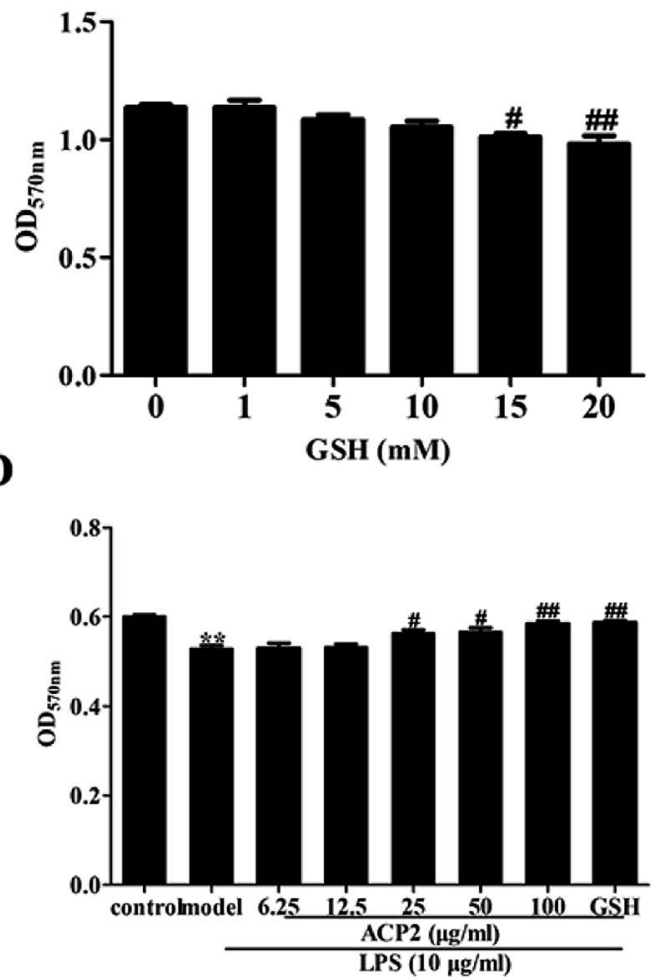

Fig. 3 Cell viability of ACP2 on L02 cells induced by LPS. MTT assay was conducted on L02 cells which treated with different concentrations of ACP2 for $24 \mathrm{~h}$. (A) Effects of various concentrations of LPS on L02 cells. (B) Effects of various concentrations of GSH on L02 cells. (C) Effects of various concentrations of ACP2 on L02 cells. (D) The proliferation of L02 cells when treated with ACP2 and LPS ( $n=6$, ** $p<0.01$ vs. the control, ${ }^{\#} p<0.05$ and ${ }^{\# \#} p<0.01$ vs. the model).

regulate the expression of pro-inflammatory cytokines. ${ }^{31,32} \mathrm{NF}-$ $\kappa \mathrm{B}$, which is one of the most pivotal downstream targets of MyD88, it activated by two signaling pathways: the NF- $\mathrm{B}$ translocation-dependent pathway and the MAPK phosphorylation pathway. NF- $\kappa \mathrm{B}$ is a heterodimer of p50 and p65 held in the cytosol with an inactive state by the inhibitor I $\mathrm{KB}-\alpha$. NF- $\kappa \mathrm{B}$ is activated and translocated to the nucleus via phosphorylation followed by the degradation of I $\kappa$ B- $\alpha .{ }^{33}$ p38, a member of MAPK family, regulates the transcription of NF- $\kappa \mathrm{B}$ and contributes to the up-regulation of some pro-inflammatory factors due to considered as an upstream mediators kinase of NF- $\kappa \mathrm{B}^{34}$ Therefore, we examined whether ACP2 regulated the expression of proteins related to MAPKs and the NF- $\kappa \mathrm{B}$ signaling pathway. First, we assessed the protein levels of I $\mathrm{B}-\alpha$ and NF- $\kappa$ B. The results indicated that LPS dramatically inhibited the level of I $\kappa \mathrm{B}-\alpha$ and increased the level of NF- $\kappa \mathrm{B}$. Meanwhile, ACP2 at the concentration of $100 \mu \mathrm{g} \mathrm{mL} \mathrm{m}^{-1}$ attenuated the up-regulation of NF- $\kappa \mathrm{B}$ and promoted I $\kappa \mathrm{B}-\alpha$ (Fig. $4 \mathrm{D}$ and $\mathrm{E} ;{ }^{\# \#} p<0.01$ ).

\subsection{ACP2 regulated MAPKs signal pathways}

Next, we evaluated the protein levels of p38, p-p38, JNK, pJNK, ERK and p-ERK. As shown in Fig. 4F-H, ACP2 at the concentration of $25 \mu \mathrm{g} \mathrm{mL}{ }^{-1}$ attenuated the levels of p-p38/ p38, which were up-regulated by LPS $\left({ }^{\# \#} p<0.01\right)$. At a concentration of $100 \mu \mathrm{g} \mathrm{mL} \mathrm{m}^{-1}$, ACP2 down-regulated p-
JNK/JNK $\left({ }^{\#} p<0.05\right)$ but had no significant regulatory effect on the expression of p-ERK/ERK. These results confirm that ACP2 exhibited an anti-inflammation effect in L02 cells, mainly through its effect on the p38/NF- $\kappa$ B signaling pathway.

\subsection{ACP2 down-regulated LPS-induced inflammation- related proteins}

The NF- $\kappa$ B and p38 signaling pathways were involved in the regulation of LPS-induced IL-1 $\beta$ and TNF- $\alpha .{ }^{33}$ Previous studies indicated that NF- $\kappa \mathrm{B}$ activation and MAPK phosphorylation are pre-conditions for the production of inflammation-related factors, including IL-1 $\beta$, COX-2, TNF- $\alpha$ and IL-6. ${ }^{35,36}$ Notably, TNF- $\alpha$ and IL-1 $\beta$ were released by some immune cells and contributed to the expression of cytokines, particularly IL-6, which was produced via macrophages and then induced an inflammatory response when exposed to LPS in the liver. Furthermore, COX-2, which is one inducible isoform of cyclooxygenase, is considered as response to miscellaneous inflammatory stimuli such as LPS. $^{37}$ Therefore, to determine the protective effect of ACP2 against LPS-induced damage to L02 cells, we measured the expressions of a series of mediators, including IL-1 $\beta$, COX-2, TNF- $\alpha$, and IL-6. Compared to the control group, the expressions of these factors were significantly increased after treatment with LPS (Fig. 4I-M), and ACP2 
A

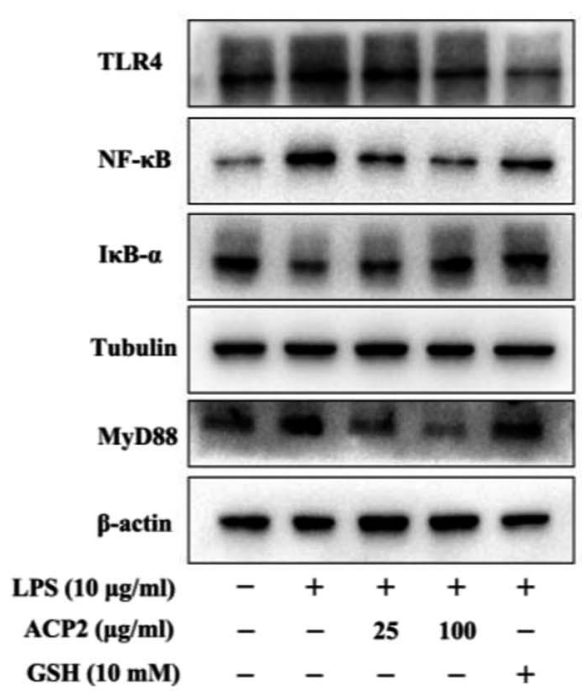

F

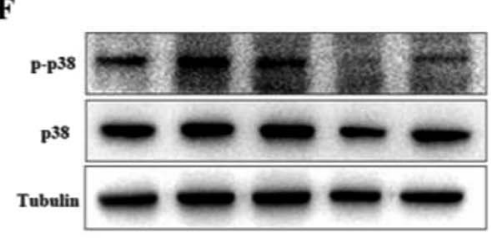

p-p38/p38

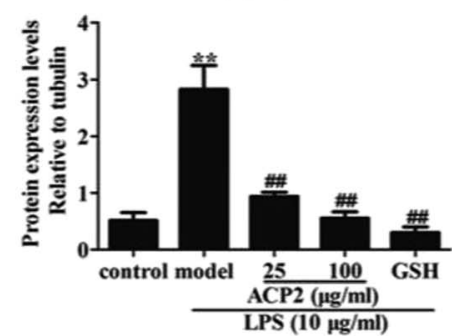

I

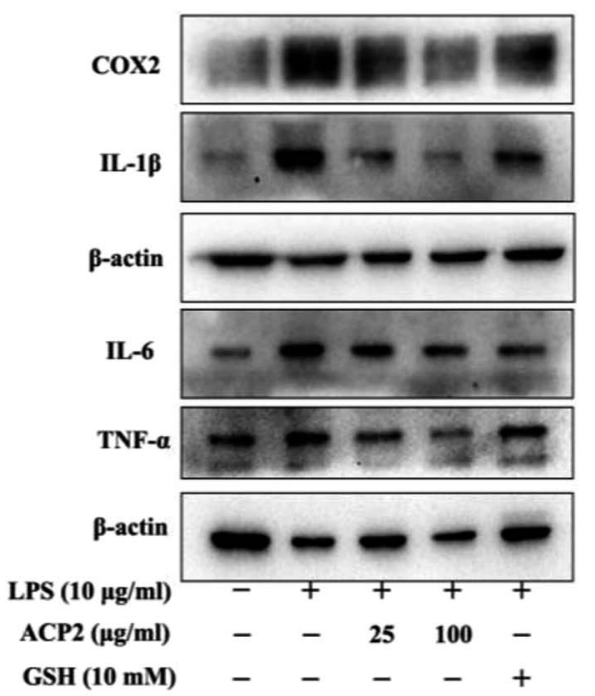

B

TLR4

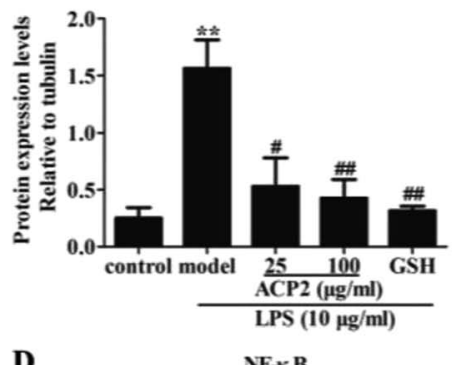

D

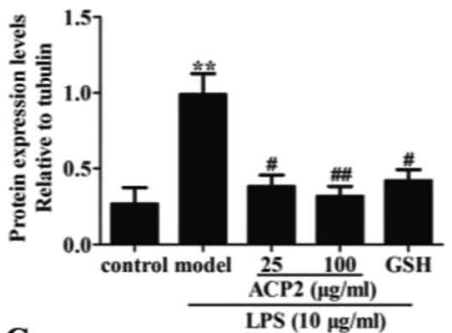

G

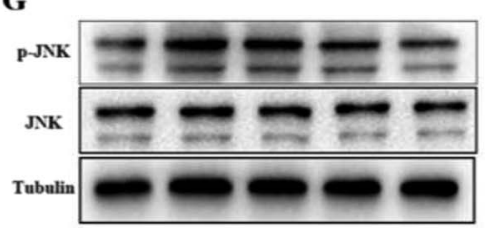

p-JNK/JNK

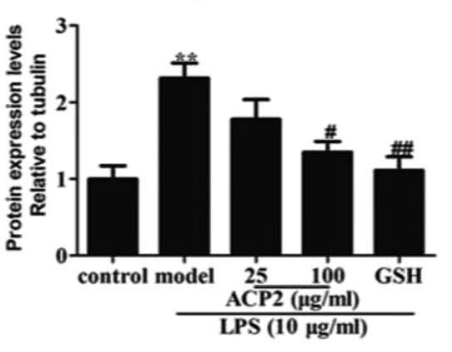

J

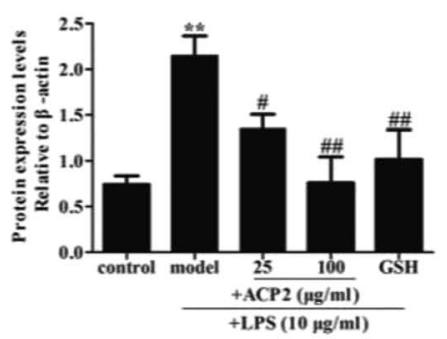

$\mathbf{L}$

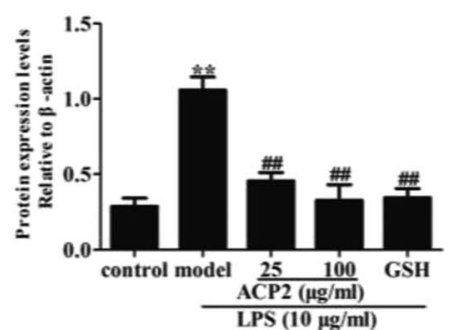

C

MyD88

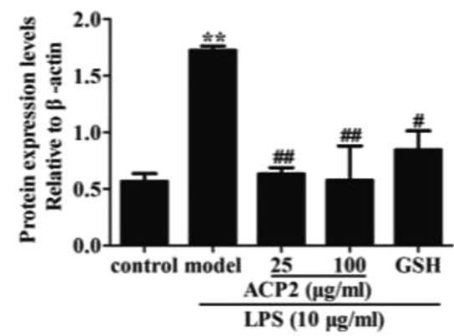

E

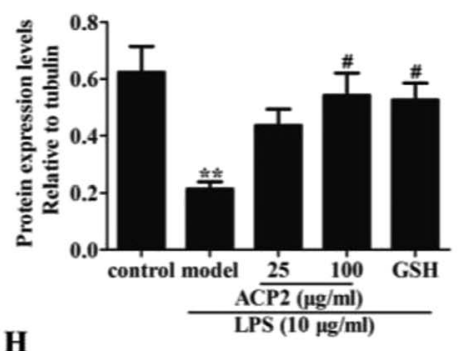

H

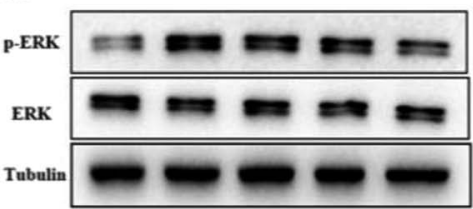

p-ERK/ERK

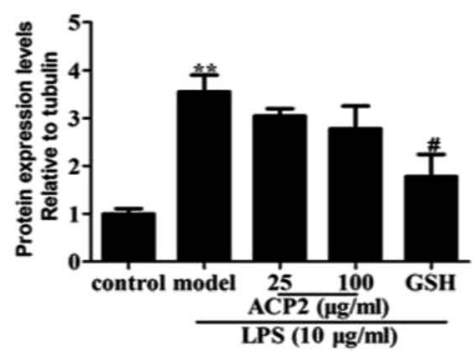

K $\quad$ L-1

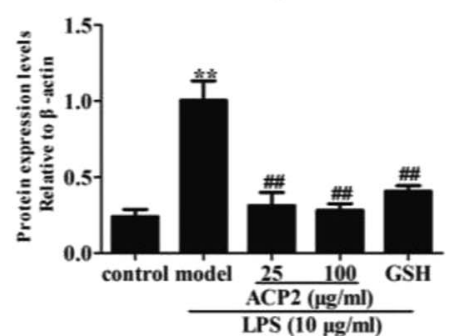

M

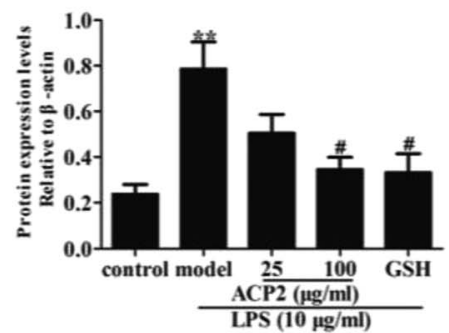

Fig. 4 (A) ACP2 attenuates the up-regulation of protein expression by LPS in L02 cells. Columns of the protein expression levels of TLR4 (B), MyD88 (C), NF-KB (D), IKB- $\alpha$ (E). ACP2 down-regulated the expression of protein associated with MAPKs. The p-p38 (F), p-JNK (G), p-ERK (H) expression levels which was standardized by tubulin. (I) The protein levels of COX-2 (J), IL-1 $\beta$ (K), TNF- $\alpha$ (L), IL-6 (M). $(n=3, * p<0.05$ and $* * p<$ 0.01 vs. the control, $\# p<0.05$ and ${ }^{\# \#} p<0.01$ vs. the model). 
attenuated these increases, in agreement with a previous study. ${ }^{38}$ Therefore, ACP2 exhibited a protective effect by downregulating inflammation-related proteins.

\section{Conclusion}

In the present study, ACP2, a galactoglucan from the mycelium of cultured A. camphorata with 6-deoxyglucose in the sugar chain, was reported for the first time. Purified, homogeneous ACP2 was water soluble and had a molecular weight of $1.93 \times$ $10^{4}$ Da. Based on by monosaccharide composition analysis, ACP2 consisted mainly of glucose, galactose and 6-deoxyglucose in a molar ratio of $5: 2: 1$. The backbone of ACP2 had the following composition: $\rightarrow 6)$-linked- $\beta$-D-Gal $p-(1 \rightarrow, \rightarrow 6)$ linked- $\alpha$-D-Gcl $p$ - $(1 \rightarrow, \rightarrow 3)$-linked- $\alpha$-D-Glc $p-(1 \rightarrow$, and $\rightarrow 2,4)$ linked- $\beta$-D-Glc $p$ - $(1 \rightarrow$, with terminal 6-deoxy- $\alpha$-D-Glc $p$ and $\alpha$-DGlc $p$. In tests with L02 cells, ACP2 exhibited protective effects by attenuating the expressions of IL- $1 \beta$, COX- 2 , TNF- $\alpha$ and IL6. ACP2 achieved these anti-inflammatory effects through the regulation of the TLR4-MyD88 signaling pathway by downregulating the expression of proteins associated with the MAPK and NF- $\kappa$ B signaling pathways. In summary, the results indicate that ACP2 can attenuate liver inflammation by downregulating the expressions of inflammation-related factors via the TLR4 receptor and $\mathrm{p} 38 / \mathrm{NF}-\kappa \mathrm{B}$ signaling pathways. The relationship between the anti-inflammatory activity and monosaccharide composition or glycosidic linkage $(e . g$., the existence of 6-deoxyglucose) should be discussed in a further study.

\section{Conflicts of interest}

The authors confirm that there are no conflicts of interest.

\section{Acknowledgements}

This work was supported by the National Natural Science Foundation of China (No. 81773985), "Double-First-Class" University project (CPU2018GF/GY16), and the 2018 Fundamental Research Funds for the Central Universities (No. 2632018ZD06). The project was funded by the Priority Academic Program Development of Jiangsu Higher Education Institutions (PAPD).

\section{References}

1 O. Krenkel and F. Tacke, Nat. Rev. Immunol., 2017, 17, 306321.

2 W. Yu, Z. Pan, Y. Zhu, F. An and Y. Lu, Eur. J. Pharmacol., 2017, 812, 234-242.

3 K. Hamesch, E. Borkham-Kamphorst, P. Strnad and R. Weiskirchen, Lab. Anim., 2015, 49, 37-46.

4 W. Lee, M. H. Hwang, Y. Lee and J. S. Bae, Chem.-Biol. Interact., 2018, 281, 106-110.

5 S. T. Anderson, S. Commins, P. N. Moynagh and A. N. Coogan, Brain, Behav., Immun., 2015, 43, 98-109.
6 X. Xia, J. Fu, X. Song, Q. Shi, C. Su, E. Song and Y. Song, Free Radicals Biol. Med., 2015, 89, 522-532.

7 L. A. Orci, S. Lacotte, V. Delaune, F. Slits, G. Oldani, V. Lazarevic, C. Rossetti, L. Rubbia-Brandt, P. Morel and C. Toso, J. Hepatol., 2018, 68, 978-985.

8 S. Y. Park, M. L. Jin, Y. H. Kim, Y. Kim and S. J. Lee, Int. Immunopharmacol., 2012, 14, 13-20.

9 Z. Rasheed and T. M. Haqqi, Biochim. Biophys. Acta, 2012, 1823, 2179-2189.

10 D. Cao, J. Luo, D. Chen, H. Xu, H. Shi, X. Jing and W. Zang, Sci. Rep., 2016, 6, 23132.

11 Y. Li, L. Chen, C. Wang, et al., Am. J. Transl. Res., 2016, 8, 5619-5627.

12 J. Xue, F. Chen, J. Wang, S. Wu, M. Zheng, H. Zhu, Y. Liu, J. He and Z. Chen, Cell. Physiol. Biochem., 2015, 35, 15571570 .

13 J. J. Cheng, C. H. Chao, P. C. Chang and M. K. Lu, Food Hydrocolloids, 2016, 53, 37-45.

14 G. J. Huang, J. S. Deng, S. S. Huang, Y. Y. Shao, C. C. Chen and Y. H. Kuo, Food Chem., 2012, 132, 709-716.

15 Z. M. Lu, W. Y. Tao, H. Y. Xu, Z. H. Ao, X. M. Zhang and Z. H. Xu, Nat. Prod. Res., 2011, 25, 684-695.

16 H. W. Chiu and K. F. Hua, PLoS One, 2016, 11, e0153087.

17 L. M. Meng, M. H. Pai, J. J. Liu and S. L. Yeh, Nutrition, 2012, 28, 942-949.

18 Q. Chen, H. Tang, Z. Zha, H. Yin, Y. Wang, Y. Wang, H. Li and L. Yue, Int. J. Biol. Macromol., 2017, 104, 768-777.

19 M. Wang, X. B. Yang, J. W. Zhao, C. J. Lu and W. Zhu, Carbohydr. Polym., 2017, 156, 390-402.

20 H. Tang, W. Wei, W. Wang, Z. Zha, T. Li, Z. Zhang, C. Luo, H. Yin, F. Huang and Y. Wang, Carbohydr. Polym., 2017, 163, 43-53.

21 K. Fukuda, T. Uematsu, A. Hamada and S. Akiya, Chem. Pharm. Bull., 1975, 23, 1955-1959.

22 I. Ciucanu and F. Kerek, Carbohydr. Res., 1984, 131, 209-217.

23 X. Xu, L. Yang, X. Xu, X. Wang, X. Chen, Q. Liang, J. Zeng and X. Jing, J. Controlled Release, 2005, 108, 33-42.

24 J. Chokboribal, W. Tachaboonyakiat, P. Sangvanich, V. Ruangpornvisuti, S. Jettanacheawchankit and P. Thunyakitpisal, Carbohydr. Polym., 2015, 133, 556566.

25 W. Liu, Y. Liu, R. Zhu, J. Yu, W. Lu, C. Pan, W. Yao and X. Gao, Carbohydr. Polym., 2016, 147, 114-124.

26 M. X. Yan, W. J. Mao, X. Liu, S. Y. Wang, Z. Xia, S. J. Cao, J. Li, L. Qin and H. L. Xian, Carbohydr. Polym., 2016, 147, 272-281. 27 P. K. Agrawal, Phytochemistry, 1992, 31, 3307-3330.

28 L. A. Possamai, M. R. Thursz, J. A. Wendon and C. G. Antoniades, J. Hepatol., 2014, 61, 439-445.

29 D. H. Kim and T. R. Billiar, J. Korean Surg. Soc., 2011, 80, 194203.

30 L. Sen, Y. Qing, Z. Qi, et al., J. Neuroinflammation, 2012, 9, 214.

31 S. K. Ha, E. Moon, M. S. Ju, D. H. Kim, J. H. Ryu, M. S. Oh and S. Y. Kim, Neuropharmacology, 2012, 63, 211-223.

32 S. W. A. Himaya, B. Ryu, Z.-J. Qian and S.-K. Kim, Toxicol. In Vitro, 2012, 26, 878-887. 
33 P. C. Liao, L. K. Chao, J. C. Chou, W. C. Dong, C. N. Lin, C. Y. Lin, A. Chen, S. M. Ka, C. L. Ho and K. F. Hua, Inflammation Res., 2012, 62, 89-96.

34 W. B. Song, Y. Y. Wang, F. S. Meng, Q. H. Zhang, J. Y. Zeng, L. P. Xiao, X. P. Yu, D. D. Peng, L. Su, B. Xiao and Z. S. Zhang, PLoS One, 2010, 5, e12969.

35 Y. Q. Chen, L. Rong and J. O. Qiao, Mol. Med. Rep., 2014, 10, 1400-1408.
36 M. S. Yoo, J. S. Shin, H. E. Choi, Y. W. Cho, M. H. Bang, N. I. Baek and K. T. Lee, Food Chem., 2012, 135, 967-975.

37 C. K. Khatri, K. S. Indalkar, C. R. Patil, S. N. Goyal and G. U. Chaturbhuj, Bioorg. Med. Chem. Lett., 2017, 27, 17211726.

38 D. K. Park and H. J. Park, BMC Complementary Altern. Med., 2013, 2013, 914524. 